\title{
Optimizing Precision of Self-Localization in the Simulated Robotics Soccer
}

\author{
Vadim Kyrylov, David Brokenshire, and Eddie Hou \\ Simon Fraser University - Surrey, \\ Surrey, British Columbia V3T 2W1 Canada \\ \{vkyrylov, brokenshire, ehou\} @sfu.ca \\ http: //www.surrey.sfu.ca/
}

\begin{abstract}
We show that previously published visual data processing methods for the simulated robotic soccer so far have not been utilizing all available information, because they were mainly based on heuristic considerations. Researchers have approached to estimating the agent location and orientation as two separate tasks, which caused systematic errors in the angular measurements. Further attempts to get rid of them (e.g. by completely neglecting the angular data) only aggravated the problem and resulted in the losses in the accuracy. We utilize all the potential of the visual sensor by jointly estimating the agent view direction angle and Cartesian coordinates using the extended Kalman filtering technique. Our experiments showed that the achievable average error limit for this particular application is about 25-33 per cent lower than that of the best algorithms published by far.
\end{abstract}

\section{Introduction}

We started this work in 2001, developing the SFUNLEASHED team for the simulated robotic soccer competitions. While trying to improve the BASIC UVA team [2, 5] used as a prototype, we realized that better, or maybe best possible, utilization of the information provided by the visual sensor was important. Player self-localization is one of related tasks.

The visual sensor of the simulated soccer agent returns measurements of the polar coordinates of objects, i.e. the range and the direction, with regular time intervals. The range to the landmarks used for self-localization (borderlines and flags) is measured relative to current agent location in the field, and the direction is measured relative to the agent neck orientation. The precise data are distorted by rounding errors. With current Soccer Server settings, quantization of the angular measurements results in the random error uniformly distributed within [-0.5, 0.5] degrees. The magnitude of the landmark range measurement error is [-0.5\%, 0.5\%] of the distance [3].

By far, processing visual sensor information with the purpose of self-localization has been addressed in different ways in the simulated robotic soccer community. The related works could be split into two groups, elaborating either on the deterministic or stochastic approaches. 


\subsection{Deterministic Methods}

Indeed, for given agent position, the visual sensor in the simulated soccer always supplies deterministic data. This feature has recently given birth to a method based on two-dimensional interval arithmetic, implemented in the LUCKY LÜBECK simulated soccer team [4]. However, because such methods are risk-aversive, the interval estimate tends to have an exaggerated size of the area to which the observed object is believed to belong. It is also unknown if this method provides the best possible accuracy, since there is just no explicitly stated optimality criteria.

One more recent work done at the Humboldt University of Berlin, uses the optimization of the mismatch between the readings of several landmarks using a gradient descent function [1]. This method offers a mathematically elegant solution, which has resulted in significantly higher precision than some simple heuristic algorithms. However, the weak point is that the angular measurements are being completely ignored in this implementation, which means that all the potential of the simulated visual sensor has not been completely utilized.

\subsection{Stochastic Methods}

Subtle notions of using stochastic estimation methods are spread over all the RoboCup literature. However, very few, if any, comprehensive overviews have been published. One early exception is the detailed description of the visual sensor data processing algorithm in [6]. For self-localization, the agent neck direction angle is estimated first. Then a weighted sum of the individual estimates of the agent position relative to different flags is used. The weights are inversely proportional to the distances from the agent to these landmarks.

A rather thorough investigation of several methods can be found in [2]. In this work, the so-called particle filter has been selected for visual sensor data processing over two simple heuristic methods and the classic Kalman filter. This method was implemented in the BASIC UVA soccer team [5], which we are using as a prototype. Compared to the nearest-flag method for agent self-localization, the particle filter reportedly yielded almost three times better accuracy. The downside was the two order of magnitude increase in the execution time required to obtain this gain. With this reservation, it looks like using the particle filter for performing the agent selflocalization task is overkill, and the grounds on which less resource-demanding Kalman filter had been rejected are not all convincing.

\subsection{Previously Mistreated Factor: The Agent Neck Direction Error}

All deterministic and stochastic methods published by far result in that the agent neck direction is estimated separately. This adds a systematic component $\delta$ to all angular measurements of the landmarks used for determining the Cartesian coordinates (Fig.1).

In Fig. 1 precise directions are shown as thin lines, while the thicker lines show the measurements distorted by the systematic error. With the self-localization algorithms proposed in $[2,4,5,6]$, the estimated agent location would be somewhere close to the center of the shaded triangle, which even does not cover the true agent location $\mathbf{A}$. Adding more flags would hardly improve the situation, because each angular measurement is equally distorted by $\delta$. We believe that this was exactly the reason of why 
some authors were reluctant about using angular data in self-localization and were relying on the range measurements only in the simulated soccer.

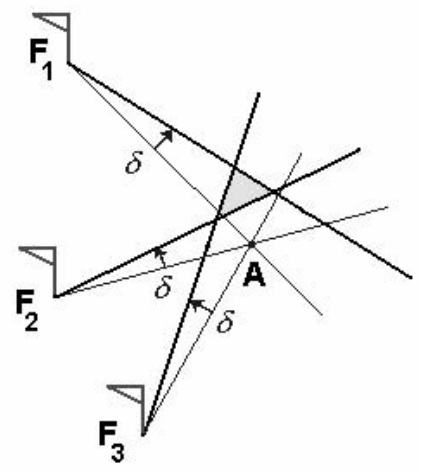

Fig. 1. The ambiguity area with the systematic error $\delta$ added to the angular measurements

This allows us to conclude that not all the potential gain has been obtained from the application of stochastic methods to visual sensor data processing in the simulated soccer. It is still unclear, where the limit of the perfection lies and how much could be in principle obtained by improving the data processing algorithms.

The purpose of this paper is two-fold. Firstly, we want to synthesize near-optimal algorithms for estimating object locations by a soccer agent completely utilizing all the potential information in the multiple readings received in one cycle. Secondly, we want to find the limits of perfection for visual sensor data processing methods in the simulated robotic soccer.

We are pursuing these goals by a comprehensive stochastic analysis of the visual sensor of the simulated soccer player. Here we consider the static case only and deliberately do not use filtering data over time, leaving the multi-cycle case for the future. Because we are using the extended Kalman filtering technique, it can be generalized for the multi-cycle case rather easily.

\section{Using the Extended Kalman Filtering Algorithm}

The complete utilization of the information supplied by the visual sensor could be achieved by jointly estimating the neck direction angle with the agent Cartesian coordinates using the extended Kalman filter [7]. It is recursively yielding an optimal estimate of the state vector $\mathbf{x}$ based on a set of measurements. The optimality criterion is the maximal likelihood of $\hat{\mathbf{x}}_{k}$ [8].

Assuming that the filter has been already applied $(k-1)$ times, on the $k$-th step its input parameters are, the measurement vector $\hat{\mathbf{z}}_{k}$ and its error covariance matrix $\mathbf{R}_{k}$. Other inputs also are the optimal state vector estimate obtained on the previous step $\hat{\mathbf{x}}_{k-1}$ and its covariance matrix $\mathbf{P}_{k-1}$. The filter returns an optimal estimate $\hat{\mathbf{x}}_{k}$ of the 
state vector and its covariance matrix $\mathbf{P}_{k}$ on current $k$-th step. In our context, $k$ is the index of the source providing raw data at the same instant of time. It is assumed that the state vector is changing with $k$ as

$$
\mathbf{x}_{\mathrm{k}}=\mathbf{f}\left(\mathbf{x}_{\mathrm{k}-1}, \mathbf{w}_{\mathrm{k}-1}\right) \text {, }
$$

where $\mathbf{f}$ is the state transition function, and $\mathbf{w}$ is the white Gaussian random sequence having zero mean and covariance matrix $\mathbf{Q}_{\mathrm{k}}$.

The measurement model has the following form:

$$
\hat{\mathbf{z}}_{\mathbf{k}}=\mathbf{h}\left(\mathbf{x}_{\mathbf{k}}, \mathbf{v}_{\mathbf{k}}\right) \text {, }
$$

where $\mathbf{h}$ is the observation transformation function and $\mathbf{v}$ is a white Gaussian measurement noise having zero mean and covariance matrix $\mathbf{R}_{\mathrm{k}}$.

The Kalman filtering algorithm is executed in three steps. First, the a priori estimate $\widetilde{\mathbf{x}}_{k}$ of the state variable vector and its covariance matrix $\widetilde{\mathbf{P}}_{k}$ are computed using (1):

$$
\begin{gathered}
\tilde{\mathbf{x}}_{\mathbf{k}}=\mathbf{f}\left(\hat{\mathbf{x}}_{\mathbf{k}-1}, 0\right), \\
\tilde{\mathbf{P}}_{\mathbf{k}}=\mathbf{A}_{\mathbf{k}} \mathbf{P}_{\mathbf{k}-1} \mathbf{A}_{\mathbf{k}}^{\mathbf{T}}+\mathbf{W}_{\mathbf{k}} \mathbf{Q}_{\mathbf{k}-1} \mathbf{W}_{\mathbf{k}}^{\mathbf{T}},
\end{gathered}
$$

where $\mathbf{A}_{\mathrm{k}}$, and $\mathbf{W}_{\mathrm{k}}$ are the Jacobian matrices [9]:

$$
A_{[i, j]}=\frac{\partial f_{[i]}}{\partial x_{[j]}}\left(\hat{x}_{k-1}, 0\right), \quad W_{[i, j]}=\frac{\partial f_{[i]}}{\partial w_{[j]}}\left(\hat{x}_{k-1}, 0\right) .
$$

Second, the Kalman matrix 'weight' is calculated:

$$
\mathbf{K}_{\mathrm{k}}=\widetilde{\mathbf{P}}_{\mathrm{k}} \mathbf{H}_{\mathrm{k}}^{\mathrm{T}}\left(\mathbf{H}_{\mathrm{k}} \widetilde{\mathbf{P}}_{\mathrm{k}} \mathbf{H}_{\mathrm{k}}^{\mathrm{T}}+\mathbf{V}_{\mathrm{k}} \mathbf{R}_{\mathrm{k}} \mathbf{V}_{\mathrm{k}}^{\mathbf{T}}\right)^{-\mathbf{1}}
$$

where $\mathbf{H}_{\mathrm{k}}$, and $\mathbf{V}_{\mathrm{k}}$ are the Jacobian matrices:

$$
H_{[i, j]}=\frac{\partial h_{[i]}}{\partial x_{[j]}}\left(\tilde{x}_{k}, 0\right), \quad V_{[i, j]}=\frac{\partial h_{[i]}}{\partial w_{[j]}}\left(\tilde{x}_{k}, 0\right) .
$$

Third, the optimal state variable vector estimate and its covariance matrix are computed:

$$
\begin{gathered}
\hat{\mathbf{x}}_{\mathrm{k}}=\widetilde{\mathbf{x}}_{\mathbf{k}}+\mathbf{K}_{\mathrm{k}}\left(\hat{\mathbf{z}}_{\mathrm{k}}-\mathbf{h}\left(\widetilde{\mathbf{x}}_{\mathrm{k}}, \mathbf{0}\right)\right), \\
\mathbf{P}_{\mathrm{k}}=\left(\mathbf{I}-\mathbf{K}_{\mathrm{k}} \mathbf{H}_{\mathrm{k}}\right) \widetilde{\mathbf{P}}_{\mathrm{k}} .
\end{gathered}
$$

Noteworthy that this estimate is only optimal when the error statistics is Gaussian. In Section 3 below we analyze how non-Gaussian statistics in the simulated soccer affects the accuracy.

Now we customize the general Kalman filtering algorithm to the particular case of estimating the simulated soccer agent state vector. For the purpose of the selflocalization, the state vector variable contains two agent Cartesian coordinates, $x, y$ and the neck direction angle, $\beta$ : 


$$
\mathbf{x}=\left[\begin{array}{lll}
x & y & \beta
\end{array}\right]^{T} .
$$

Now consider the model of the state vector dynamics (1). Because we are dealing with a snapshot, this allows us to reduce (3) to the trivial linear model

$$
\mathbf{x}_{\mathbf{k}}=\mathbf{x}_{\mathrm{k}-1} \text {. }
$$

Moreover, the Jacobian matrices $\mathbf{A}_{\mathrm{k}}$, and $\mathbf{W}_{\mathrm{k}}$ in (4) are both zero matrices and we get:

$$
\widetilde{\mathbf{P}}_{\mathbf{k}}=\mathbf{P}_{\mathbf{k}-1},
$$

The measurement model (2) is substantially nonlinear. The observation vector for $k$-th landmark is the pair of polar coordinates, with the direction (azimuth) $d_{k}$ measured relative to the agent neck direction and the range $r_{k}$ measured from the agent to the landmark:

$$
\mathbf{z}_{k}=\left[\begin{array}{ll}
r_{k} & d_{k}
\end{array}\right]^{T}
$$

From the geometry of the relationship between $\mathbf{z}$ and $\mathbf{x}$ we get:

$$
\mathbf{z}_{\mathbf{k}}=\mathbf{h}_{\mathbf{k}}\left(\mathbf{x}, \mathbf{v}_{\mathbf{k}}\right)
$$

where

$$
\begin{aligned}
& r_{k}=h_{k}^{(1)}\left(\mathbf{x}, \mathbf{v}_{\mathbf{k}}\right)=\sqrt{\left(x-x_{F k}\right)^{2}+\left(y-y_{F k}\right)^{2}}+v_{r k}, \\
& d_{k}=h_{k}^{(2)}\left(\mathbf{x}, \mathbf{v}_{\mathbf{k}}\right)=\tan ^{-1}\left(\frac{y-y_{F k}}{x-x_{F k}}\right)-\beta+v_{d k},
\end{aligned}
$$

$x_{F k}, y_{F k}$ are the Cartesian coordinates of the $k$-th reference point $\mathbf{F}_{k}$, and $\mathbf{v}_{\mathbf{k}}=\left[\begin{array}{ll}v_{r k} & v_{d k}\end{array}\right]^{T}$ is the random measurement error vector.

The low index $k$ in (2a) underscores that, in general, for different landmarks the measurement model could be different. For all visible flags the covariance matrix is,

$$
\mathbf{R}_{k}=\left[\begin{array}{cc}
r_{k}^{2} \sigma_{r}^{2} & 0 \\
0 & \sigma_{d}^{2}
\end{array}\right],
$$

where $\sigma_{d}^{2}=1 /\left(12 \cdot\left(180^{\circ} / \pi\right)^{2}\right)$ and $\sigma_{r}^{2}=(0.01)^{2} / 12$ for static objects [3].

For the field borderlines, however, the measurement model differs in that the range measurements are unavailable and only the direction can be measured. To keep the same model for both types of landmark, in the covariance matrix (10) for borderlines we assume that $\sigma_{r}^{2}=+\infty$.

For calculating the matrix weight (5) we need two Jacobian matrices, $\mathbf{H}_{\mathrm{k}}$, and $\mathbf{V}_{\mathrm{k}}$. They immediately follow from (2a) and (10). 


$$
\begin{gathered}
\mathbf{H}_{k}=\left[\begin{array}{ccc}
\frac{x}{\sqrt{\left(x-x_{F k}\right)^{2}+\left(y-y_{F k}\right)^{2}}} & \frac{y}{\sqrt{\left(x-x_{F k}\right)^{2}+\left(y-y_{F k}\right)^{2}}} & 0 \\
-\frac{y-y_{F k}}{\left(x-x_{F k}\right)^{2}+\left(y-y_{F k}\right)^{2}} & \frac{x-x_{F k}}{\left(x-x_{F k}\right)^{2}+\left(y-y_{F k}\right)^{2}} & -1
\end{array}\right], \\
\mathbf{V}_{k}=\mathbf{V}=\left[\begin{array}{ll}
1 & 0 \\
0 & 1
\end{array}\right] .
\end{gathered}
$$

Therefore, the sought matrix weight can be calculated, as follows:

$$
\mathbf{K}_{\mathbf{k}}=\mathbf{P}_{\mathbf{k}-1} \mathbf{H}_{\mathrm{k}}^{\mathrm{T}}\left(\mathbf{H}_{\mathrm{k}} \mathbf{P}_{\mathrm{k}-1} \mathbf{H}_{\mathrm{k}}^{\mathrm{T}}+\mathbf{R}_{\mathrm{k}}\right)^{-\mathbf{1}} \text {. }
$$

This weight is used to compute the optimal state variable vector estimate

$$
\hat{\mathbf{x}}_{\mathbf{k}}=\widehat{\mathbf{x}}_{\mathbf{k}-1}+\mathbf{K}_{\mathbf{k}}\left(\hat{\mathbf{z}}_{\mathbf{k}}-\mathbf{h}_{k}\left(\hat{\mathbf{x}}_{\mathbf{k}-1}, \mathbf{0}\right)\right),
$$

and its covariance matrix

$$
\mathbf{P}_{\mathbf{k}}=\left(\mathbf{I}-\mathbf{K}_{\mathbf{k}} \mathbf{H}_{\mathbf{k}}\right) \mathbf{P}_{\mathbf{k}-1} .
$$

Expressions (3a-7a), coupled with (10-12) yield the sought optimal algorithm for agent localization in the simulated soccer. For $N$ visible landmarks it must be executed for $k=1,2, \ldots, N$.

The initial state $\left(\hat{\mathbf{x}}_{\mathbf{0}}, \mathbf{P}_{\mathbf{0}}\right)$ for this algorithm is extrapolated from the previous $(k-1)$ th cycle, which increases the self-localization error 2..3 times. With more than three flags visible on $k$-th cycle, this allows ignoring measurements made in the previous cycle and just set

$$
\mathbf{P}_{0}=\left[\begin{array}{ccc}
+\infty & 0 & 0 \\
0 & +\infty & 0 \\
0 & 0 & +\infty
\end{array}\right]
$$

This results in that (5a) assigns very large weight to the innovation in (6a) and we do not care about $\hat{\mathbf{x}}_{0}$ at all. However, less than three flags are visible 9 per cent time for 90-degree visual sector and 1 per cent time for 180-degrees. Because ignoring history data in these cases would result in the increased errors, the diagonal elements in $\mathbf{P}_{0}$ are set to finite positive values.

\section{Simulation Results}

To determine the precision of the proposed method, we have run five sets of simulations. Each of them contained total 2000 experiments by placing the agent in the random points which were uniformly spread over the field. The neck direction was uniformly distributed in the $\left[0^{\circ}, 360^{\circ}\right)$ interval. The agent viewing sector was either 90 or 180 degrees wide, with average number of visible flags 5.7 and 11.4, respectively. The results (Table 1) are accurate with less than $\pm 0.9 \%$ error, 19 times of 20 . 
Experiment \#1 estimates the achievable accuracy of the proposed algorithm and provides a benchmark for comparing it with the alternative self-localization methods. Experiment \#2 estimated the loss in precision which resulted from violating the assumption that the visual sensor measurement errors are Gaussian as required by Kalman filter theory We compared the self-localization errors in two cases: (a) default Soccer Server rounding errors $\left( \pm 0.5^{\circ}\right.$ for the angle and $\pm 0.5 \%$ for the range), and (b) Gaussian errors having zero means and same standard deviations (i.e. $0.289^{\circ}$ and $0.289 \%$, respectively).

Table 1. Experiments with the self-localization algorithms (90-dergee agent viewing sector)

\begin{tabular}{|c|l|l|l|}
\hline $\begin{array}{l}\text { Experi } \\
\text { ment }\end{array}$ & Description & Objective & $\begin{array}{l}\text { Localization } \\
\text { error, meters }\end{array}$ \\
\hline 1 & $\begin{array}{l}\text { Kalman Filter as proposed } \\
\text { in Section 2 }\end{array}$ & $\begin{array}{l}\text { Estimating absolute accu- } \\
\text { racy }\end{array}$ & $0.091(100 \%)$ \\
\hline 2 & $\begin{array}{l}\text { Kalman Filter with Gaus- } \\
\text { sian measurement error } \\
\text { statistics }\end{array}$ & $\begin{array}{l}\text { Estimating loss of preci- } \\
\text { sion from non-Gaussian } \\
\text { statistics }\end{array}$ & $0.090(98.9 \%)$ \\
\hline 3 & $\begin{array}{l}\text { Kalman Filter with ignored } \\
\text { angular measurements }\end{array}$ & $\begin{array}{l}\text { Estimating the gain from } \\
\text { using angular data }\end{array}$ & $0.13(143 \%)$ \\
\hline 4 & $\begin{array}{l}\text { All-flags, with systematic } \\
\text { angular error present }\end{array}$ & $\begin{array}{l}\text { Comparison with the } \\
\text { widely used algorithm }\end{array}$ & $0.11(121 \%)$ \\
\hline 5 & $\begin{array}{l}\text { Nearest flag algorithm } \\
\text { Comparison with the } \\
\text { simplest method }\end{array}$ & $0.30(330 \%)$ \\
\hline
\end{tabular}

We found that in the Gaussian case, for which the Kalman filter is strictly optimal, the mean error is $98.9 \%$ of the error obtained in the default case. This gives the idea of the price paid for the sub optimality. Since the difference is very close to the accuracy limit of our experiments, it is clear that the non-Gaussian statistics only insignificantly affects the estimator quality. Presumably this unreasonable concern was the main reason for using particle filter in [2].

Experiment \#3 evaluated the loss in precision when angular measurements are ignored, as suggested by some of our predecessors. The test was run by setting in (10) the angular variance $\sigma_{d}^{2} 1000$ times greater that it originally was. This forced the Kalman filter to ignore angular measurements and resulted in a $43 \%$ increase of the error.

Experiment \#4 evaluates a very common self-localization algorithm. It is using one border line for estimating the neck direction angle and then is utilizing all visible flags to estimate the agent location. No care is taken of the systematic angular error, like shown in Fig.1. Although this algorithm has been developed by us, it is combining the ideas, which are widely spread in the simulated soccer community. We speculate that it could be somewhat more accurate than each of its predecessors, because it is taking correlation of the Cartesian coordinates measurements into account. Still compared to the Kalman filter, it results in the self-localization error which is greater 
by $21 \%$. We have also run similar experiment with a 180 degree visual sector, which resulted in a $45 \%$ increase of the error. Assuming that 90- and 180-degree sectors are being used with equal probability, the average localization error would be greater by $33 \%$.

Experiment \#5 gives the reference point for the achievable accuracy of the proposed method as compared to the simplest, the nearest-flag self-localization algorithm. The Kalman filter offers a three-fold accuracy gain in this case. This is consistent with the reported gain from using the particle filter [2].

It is safe to say that Table 1 contains the minimal achievable average selflocalization errors in the simulated soccer and could be used as the reference point.

In a separate set of the experiments we assessed the benefits of two-agent ball tracking. Two agents were simultaneously viewing the ball from different locations. Each agent knew its location and orientation precisely, and could relay his estimate of the ball coordinates to the second agent without increasing the estimation error. The latter agent was using two sets of ball $(x, y)$ coordinate measurements to estimate the ball location more precisely. Noteworthy that, unlike landmarks, ball Cartesian coordinate measurement errors may be highly correlated.

We compared two algorithms, heuristic and the Kalman filter (Table 2). The first algorithm was calculating the ball Cartesian coordinates based on the range and direction measurements by the agents. Then two $x, y$ pairs of the ball coordinates were being merged into the final estimate using the near-optimal method based on the maximal-likelihood criterion [10] and its two modifications. One modification ignored the correlation between the Cartesian coordinates, and the second ignored the angular data. The Kalman filter was using the joint estimate of the ball location based on the observations of its polar coordinates by the two agents.

Agents were placed 2000 times 10 meters apart in random locations at the distance from the ball uniformly distributed in the 5 to 15 meter interval. The results are accurate with less than $\pm 1.1 \%$ error, 19 times of 20 . They show that, while estimating the ball location in a 90-degree viewing sector, the assistance by a teammate can potentially reduce the mean linear error about two times. Ignoring correlation would reduce this gain to just 1.3 times. Ignoring angular measurements would be counterproductive.

Table 2. One- and two-agent ball tracking average linear error (in meters) using different estimation algorithms (90-degree sector)

\begin{tabular}{|c|c|c|c|c|}
\hline \multirow{2}{*}{ One agent } & \multicolumn{4}{|c|}{ Two agents } \\
\cline { 2 - 5 } & Ignore angles & Ignore-correlation & Near-optimal & Kalman Filter \\
\hline $\begin{array}{c}1.17 \\
(193 \%)\end{array}$ & $4.10(676 \%)$ & $0.78(129 \%)$ & $0.606(100 \%)$ & $\begin{array}{c}0.601 \\
(99.2 \%)\end{array}$ \\
\hline
\end{tabular}

In other experiments reported elsewhere [10] we have also found that the joint estimation of the Cartesian coordinates and the agent orientation would offer more significant gains in the robotics application, where the systematic error is greater than in the simulated soccer $\left(0.5^{\circ}\right)$. In particular, we have found that the average localization error can be decreased $4 . .8$ times, if the angular bias is uniformly distributed in the interval as big as $\pm 4.0^{\circ}$. This implies that the significance of this work is not 
limited to the simulated soccer. Rather, with only minor changes the proposed algorithm can be reused in other robotics applications, where joint estimation of the robot location and its orientation could result in significant gains.

\section{Conclusion}

We have derived algorithms for determining absolute Cartesian coordinates of objects using imprecise readings of local polar coordinates, supplied by a visual sensor in a system like robotic soccer. The proposed solution is based on the Kalman filtering technique.

The innovation is in the rigorous treatment of this problem from the positions of the stochastic estimation theory. In particular, by jointly estimating the agent location and orientation, we have taken into account the correlation of the raw measurements which is emerging after converting them into Cartesian coordinate system. By far, this correlation has been neglected in the simulated soccer applications, which resulted in some losses. Although in self-localization this loss is negligible, in the related task of ball tracking using agent communication negligence of correlation increases the linear error by 30 per cent.

Using the joint estimation of the agent location and orientation allows walking around the problem of the systematic error present in the angular measurements. This problem has been persisting in the self-localization algorithms published so far and limited their accuracy. In particular, we have shown that ignoring the angular data as the way to getting rid of the systematic error would result in about 43 per cent increase in the localization error.

We have demonstrated that the non-Gaussian statistics of the raw measurement errors, which presumably was a concern for some researchers who have been reluctant about using Kalman filter in the simulated soccer on these grounds, is indeed not an obstacle. Our experiments has shown that replacing the non-Gaussian errors with the equivalent 'true' normal noise would not result in statistically significant differences in the self-localization accuracy. Compared to the best published algorithms, the new method can reduce the average error by $25-33$ per cent.

Because we have shown that the assumption made about Gaussian statistics does not reduce the precision, we can guarantee that, the solution found cannot be tangibly improved in terms of the mean error of the location estimate. We thus indeed have utilized all the potential of the visual sensor, as it applies to single simulation cycle.

The computational effort required for implementing this algorithm is proportional to the number of the landmarks used for self-localization. Compared with the nearest flag method, the increase in the computation time is roughly six-fold. We believe that it is a fair cost for gaining a three times higher accuracy than the nearest-flag algorithm.

We also have found that the proposed algorithm could potentially offer even more gains in cases when the systematic angular errors are greater that those present in the simulated soccer. Therefore we hope that after some modifications, our algorithm could be reused in some other robotics applications. 
The results, however, are limited to data processing in a single simulation cycle. Our future work will be targeted at similar comprehensive study of the coordinate data processing over time using all the power of methods offered by the Kalman filtering technique.

\section{References}

1. Bach, J., Gollin, M.: Self-Localization revisited. In: Birk, A., Coradeschi, S., Tadokoro, S. (eds.): RoboCup 2001, Lecture Notes in Artificial Intelligence, Vol. 2377. Springer-Verlag Berlin Heidelberg New York (2002) 251-256

2. de Boer, R., Kok, J.: The Incremental Development of a Synthetic Multi-Agent System: The UvA Trilearn 2001 Robotic Soccer Simulation Team. M.S. Thesis. Faculty of Science, University of Amsterdam (2002)

3. Foroughi. E., Heintz, F., Kapetanakis, K., Kotiadis, K., Kummeneje, J., Noda, I., Obst, O, Riley, P., and Steffens, T.: RoboCup Soccer Server User Manual. Versions 7.06 and later (2001) http://sourceforge.net/projects/sserver

4. Haker, M., Meyer, A., Polani, D., and Martinez, T.: A Method for Incorporation of New Evidence to Improve World State Estimation. In: Birk, A., Coradeschi, S., Tadokoro, S. (eds.): RoboCup 2001, Lecture Notes in Artificial Intelligence, Vol. 2377. Springer-Verlag Berlin Heidelberg New York (2002) 362-367

5. Kok, J., de Boer, R., Groen, F., Vlassis, N.: UvA Trilearn 2001 - Soccer Simulation Team. Faculty of Science, University of Amsterdam (2001) http://carol.wins.uva.nl/ jellekok/ robocup/2001/index_en.html

6. Müller-Gugenberger P., Wendler, J.: AT Humboldt 98 -- Design, Implentierung und Evaluierung eines Multiagentensystems für den RoboCup-98 mittels einer BDIArchitektur. Diploma thesis. Huboldt University of Berlin (1998) http://www.informatik. hu-berlin.de/ wendler/paper/gugenberger Wendler:diplom:1998.ps.gz

7. Catlin, D. (1989) Estimation, Control, and the Discrete Kalman Filter (Applied Mathematical Sciences, Vol 71) Springer Verlag, ISBN: 038796777X

8. MathWorld: Maximal Likelihood, http://mathworld.wolfram.com/Maximum Likelihood.html

9. MathWorld: Jacobian, http://mathworld.wolfram.com/Jacobian.html

10. Kyrylov, V., Brokenshire, D., Hou, E. Optimizing Precision of Self-Localization in Simulated Soccer Agents. Technical report SIAT-TR-VK-01-2003. 